\title{
New results for Brillouin electron beam focusing system
}

\section{Zhibo Cheng ${ }^{1,2^{*}}$ and Shaowen Yao ${ }^{1 *}$}

\section{"Correspondence:} czbo@hpu.edu.cn; yaoshaowen@hpu.edu.cn ${ }^{1}$ School of Mathematics and Information Science, Henan Polytechnic University, Jiaozuo, 454000, China

2Department of Mathematics, Sichuan University, Chengdu, 610064, China

\begin{abstract}
An experimental conjecture on the existence of positive periodic solutions for the Brillouin electron beam focusing system $x^{\prime \prime}+a(1+\cos 2 t) x=\frac{1}{x}$ for $a \in\left(0, \frac{1}{2}\right)$ is proved by applications of the Manasevich-Mawhin theorem.
\end{abstract}

MSC: 34K14; 34C25

Keywords: Brillouin electron beam focusing system; positive periodic solution; singular

\section{Introduction}

In this paper, we consider the $2 \pi$-periodic boundary value problem for the equation

$$
x^{\prime \prime}+a(1+\cos 2 t) x=\frac{1}{x}
$$

where $a>0$ is constant.

The equations arise in the study of electronics and govern the motion of a magnetically focused axially symmetric electron beam under the influence of a Brillouin flow [1]. When the negative pole in a traveling-wave tube is shielded completely by a magnetic field screen, the electron beam focusing system can be described by (1.1). Besides, from a mathematical point of view, equation (1.1) is a singular perturbation of the Mathieu equation.

Motivated by the results of laboratory experiments experts realized in [1], it was conjectured that (1.1) should have a positive periodic solutions if $a \in\left(0, \frac{1}{4}\right)$ [2]. In the last fifty years, many mathematicians have given birth to extensive literature about this topic (see [3-7]). Although numerical studies back up the experimental conjecture, an analytical proof of the existence of periodic solutions of (1.1) for $a \in\left(0, \frac{1}{4}\right)$ is still lacking.

The first analytic work on periodic solution of (1.1) was obtained by Ding [3]. Ding proved that (1.1) had at least one positive periodic solution if $a \in\left(0, \frac{1}{16}\right)$. Afterwards, Ye and Wang [4] obtained that (1.1) had at least one positive periodic solution if $a \in(0,0.1442)$. In [5], Zhang investigated a kind of singular Liénard equation, and by applications of his theory, they extended the existence result of (1.1) to $a \in(0,0.1532)$.

However, in the above works, authors were not able to prove or disprove the result which was conjectured in [1]. In this paper, we will show that (1.1) has at least one positive $2 \pi$ periodic solution when the parameter $a \in\left(0, \frac{1}{2}\right)$ other than $\left(0, \frac{1}{4}\right)$.

(c) The Author(s) 2017. This article is distributed under the terms of the Creative Commons Attribution 4.0 International License (http://creativecommons.org/licenses/by/4.0/), which permits unrestricted use, distribution, and reproduction in any medium, provided you give appropriate credit to the original author(s) and the source, provide a link to the Creative Commons license, and indicate if changes were made. 


\section{Brillouin electron beam focusing system}

Lemma 2.1 (Manasevich-Mawhin [8]) Let $\Omega$ be an open bounded set in $C_{T}^{1}:=\{x \in$ $\left.C^{1}(\mathbb{R}, \mathbb{R}): x(t+T)-x(t) \equiv 0\right\}$. If

(i) The problem

$$
\left(\phi\left(x^{\prime}\right)\right)^{\prime}=\lambda \tilde{f}\left(t, x, x^{\prime}\right), \quad x \in C_{T}^{1},
$$

where $\tilde{f}:[0, T] \times \mathbb{R} \times \mathbb{R} \rightarrow \mathbb{R}$ is assumed to be Carathéodory. For each $\lambda \in(0,1)$, problem (2.1) has no solution on $\partial \Omega$.

(ii) The equation

$$
F(a):=\frac{1}{T} \int_{0}^{T} \tilde{f}\left(t, x, x^{\prime}\right) d t=0,
$$

has no solution on $\partial \Omega \cap \mathbb{R}$.

(iii) The Brouwer degree of $F$

$$
\operatorname{deg}\{F, \Omega \cap \mathbb{R}, 0\} \neq 0
$$

Then problem (2.1) has at least one periodic solution on $\bar{\Omega}$.

Lemma 2.2 ([9]) Suppose that $u \in C_{T}^{1}$ and there exists $t_{0} \in[0, T]$ such that $\left|u\left(t_{0}\right)\right|<d$. Then

$$
\left(\int_{0}^{T}|u(t)|^{2} d t\right)^{\frac{1}{2}} \leq\left(\frac{T}{\pi}\right)\left(\int_{0}^{T}\left|u^{\prime}(t)\right|^{2} t\right)^{\frac{1}{2}}+d T^{\frac{1}{2}} .
$$

Next, we prove that Brillouin electron beam focusing system (1.1) has at least one positive $2 \pi$-periodic solution if $a \in\left(0, \frac{1}{2}\right)$. Firstly, we consider the following singular equation:

$$
x^{\prime \prime}(t)+a(t) x(t)=\frac{1}{x(t)},
$$

where $a(t) \in C(\mathbb{R},[0,+\infty))$ and $a(t+T)=a(t), \forall t \in \mathbb{R}$.

Theorem 2.1 Assume that $|a|_{\infty}:=\max _{t \in[0, T]}|a(t)|<\frac{4 \pi^{2}}{T^{2}}$ holds. Then (2.2) has at least one positive T-periodic solution.

Proof Firstly, we consider the following (homotopy) family of (2.2):

$$
x^{\prime \prime}(t)+\lambda\left(a(t) x(t)-\frac{1}{x(t)}\right)=0, \quad \lambda \in(0,1]
$$

Let $x(t) \in C_{T}^{1}$ be an arbitrary solution of (2.3). Integrating (2.3) from 0 to $T$, we get

$$
\int_{0}^{T}\left(a(t) x(t)-\frac{1}{x(t)}\right) d t=0
$$

So, we know that there exist positive constants $D_{1}<D_{2}$ and $t_{0} \in(0, T)$ such that

$$
D_{1} \leq x\left(t_{0}\right) \leq D_{2}
$$


Therefore, we have

$$
|x(t)|=\left|x\left(t_{0}\right)+\int_{t_{0}}^{t} x^{\prime}(s) d s\right| \leq D_{2}+\int_{0}^{T}\left|x^{\prime}(s)\right| d s
$$

Let us write $x(t)=\bar{x}+\tilde{x}(t)$, here $\tilde{x}(t):=x(t)-\bar{x}$, and $\bar{x}:=\frac{1}{T} \int_{0}^{T} x(t) d t$. Obviously, $\int_{0}^{T} \tilde{x}(t) d t=0$. Now (2.3) for $\tilde{x}(t)$ is

$$
\tilde{x}^{\prime \prime}(t)+\lambda a(t)(\bar{x}+\tilde{x}(t))=\lambda \frac{1}{x(t)}
$$

since $\bar{x}^{\prime \prime}=0$. Multiplying $(2.7)$ by $\bar{x}-\tilde{x}(t)$, we have

$$
\bar{x} \tilde{x}^{\prime \prime}(t)-\tilde{x}(t) \tilde{x}^{\prime \prime}(t)+\lambda a(t)\left(\bar{x}^{2}-\tilde{x}^{2}(t)\right)=\lambda \frac{\bar{x}-\tilde{x}(t)}{x(t)} .
$$

Integrating this equation over one period and making use of the $T$-periodicity of $\tilde{x}(t)$, we get

$$
-\int_{0}^{T} \tilde{x}(t) \tilde{x}^{\prime \prime}(t) d t+\lambda \int_{0}^{T} a(t)\left(\bar{x}^{2}-\tilde{x}^{2}(t)\right) d t=\lambda \int_{0}^{T} \frac{\bar{x}-\tilde{x}(t)}{x(t)} d t .
$$

So, we have

$$
\int_{0}^{T}\left|\tilde{x}^{\prime}(t)\right|^{2} d t=\lambda \int_{0}^{T} a(t) \tilde{x}^{2}(t) d t-\lambda \bar{x}^{2} \int_{0}^{T} a(t) d t+\lambda \int_{0}^{T} \frac{\bar{x}-\tilde{x}(t)}{x(t)} d t .
$$

Since $a(t) \geq 0$, then $-\bar{x}^{2} \int_{0}^{T} a(t) d t \leq 0$. So, we have

$$
\begin{aligned}
\int_{0}^{T}\left|\tilde{x}^{\prime}(t)\right|^{2} d t & \leq \lambda \int_{0}^{T} a(t) \tilde{x}^{2}(t) d t+\lambda \int_{0}^{T} \frac{\bar{x}-\tilde{x}(t)}{x(t)} d t \\
& =\lambda \int_{0}^{T} a(t) \tilde{x}^{2}(t) d t+\lambda \int_{0}^{T} \frac{2 \bar{x}-x(t)}{x(t)} d t \\
& =\lambda \int_{0}^{T} a(t) \tilde{x}^{2}(t) d t+\lambda \int_{0}^{T} \frac{2 \bar{x}}{x(t)} d t-\lambda T \\
& \leq \int_{0}^{T}|a(t)||\tilde{x}(t)|^{2} d t+2|\bar{x}| \int_{0}^{T} \frac{1}{|x(t)|} d t .
\end{aligned}
$$

For any $\varepsilon>0$, there is $g_{\varepsilon}^{+} \in L^{2}(0, T)$ and $g_{\varepsilon}^{+}>0$

$$
\frac{1}{x(t)} \leq \varepsilon x(t)+g_{\varepsilon}^{+}(t)
$$

for all $x(t)>0$ and a.e. $t \in[0, T]$. So, we have

$$
\begin{aligned}
\int_{0}^{T}\left|\tilde{x}^{\prime}(t)\right|^{2} & \leq \int_{0}^{T}|a(t)||\tilde{x}(t)|^{2} d t+2 \bar{x} \int_{0}^{T}\left(\varepsilon x(t)+g_{\varepsilon}^{+}(t)\right) d t \\
& \leq \int_{0}^{T}|a(t)||\tilde{x}(t)|^{2} d t+\frac{2}{T} \int_{0}^{T}|x(t)| d t \int_{0}^{T}\left(\varepsilon x(t)+g_{\varepsilon}^{+}(t)\right) d t
\end{aligned}
$$




$$
\begin{aligned}
& \leq|a|_{\infty} \int_{0}^{T}|\tilde{x}(t)|^{2} d t+\frac{2 \varepsilon}{T}\left(\int_{0}^{T}|x(t)| d t\right)^{2}+\frac{2}{T} \int_{0}^{T}|x(t)| d t \int_{0}^{T} g_{\varepsilon}^{+}(t) d t \\
& \leq|a|_{\infty} \int_{0}^{T}|\tilde{x}(t)|^{2} d t+2 \varepsilon \int_{0}^{T}|x(t)|^{2} d t \\
& \quad+2\left(\int_{0}^{T}|x(t)|^{2} d t\right)^{\frac{1}{2}}\left(\int_{0}^{T}\left|g_{\varepsilon}^{+}(t)\right|^{2} d t\right)^{\frac{1}{2}},
\end{aligned}
$$

where $|a|_{\infty}=\max _{t \in[0, T]}|a(t)|$. Since $D_{1} \leq x\left(t_{0}\right) \leq D_{2}$, by Lemma 2.2 , we have

$$
\left(\int_{0}^{T}|x(t)|^{2} d t\right)^{\frac{1}{2}} \leq\left(\frac{T}{\pi}\right)\left(\int_{0}^{T}\left|x^{\prime}(t)\right|^{2} d t\right)^{\frac{1}{2}}+D_{2} \sqrt{T}
$$

By applications of Wirtinger's inequality (in [10] Lemma 2.4) and (2.9), we have

$$
\begin{aligned}
\int_{0}^{T}\left|\tilde{x}^{\prime}(t)\right|^{2} \leq & |a|_{\infty}\left(\frac{T}{2 \pi}\right)^{2} \int_{0}^{T}\left|\tilde{x}^{\prime}(t)\right|^{2} d t+2 \varepsilon\left(\left(\frac{T}{\pi}\right)\left(\int_{0}^{T}\left|x^{\prime}(t)\right|^{2} d t\right)^{\frac{1}{2}}+D_{2} \sqrt{T}\right)^{2} \\
& +2\left(\left(\frac{T}{\pi}\right)\left(\int_{0}^{T}\left|x^{\prime}(t)\right|^{2} d t\right)^{\frac{1}{2}}+D_{2} \sqrt{T}\right)\left\|g_{\varepsilon}^{+}\right\|_{2} \\
= & |a|_{\infty}\left(\frac{T}{2 \pi}\right)^{2} \int_{0}^{T}\left|\tilde{x}^{\prime}(t)\right|^{2} d t+2 \varepsilon\left(\frac{T}{\pi}\right)^{2} \int_{0}^{T}\left|x^{\prime}(t)\right|^{2} d t \\
& +2\left(2 D_{2} \sqrt{T} \varepsilon+\left\|g_{\varepsilon}^{+}\right\|_{2}\right)\left(\frac{T}{\pi}\right)\left(\int_{0}^{T}\left|x^{\prime}(t)\right|^{2} d t\right)^{\frac{1}{2}} \\
& +2 D_{2}^{2} T \varepsilon+2 D_{2} \sqrt{T}\left\|g_{\varepsilon}^{+}\right\|_{2}
\end{aligned}
$$

where $\left\|g_{\varepsilon}^{+}\right\|_{2}=\left(\int_{0}^{T}\left|g_{\varepsilon}^{+}(t)\right|^{2} d t\right)^{\frac{1}{2}}$. Since $\tilde{x}^{\prime}(t)=x^{\prime}(t)$, then we have

$$
\begin{aligned}
\int_{0}^{T}\left|x^{\prime}(t)\right|^{2} \leq & \left(|a|_{\infty}\left(\frac{T}{2 \pi}\right)^{2}+2 \varepsilon\left(\frac{T}{\pi}\right)^{2}\right) \int_{0}^{T}\left|x^{\prime}(t)\right|^{2} d t \\
& +2\left(2 D_{2} \sqrt{T} \varepsilon+\left\|g_{\varepsilon}^{+}\right\|_{2}\right)\left(\frac{T}{\pi}\right)\left(\int_{0}^{T}\left|x^{\prime}(t)\right|^{2} d t\right)^{\frac{1}{2}} \\
& +2 D_{2}^{2} T \varepsilon+2 D_{2} \sqrt{T}\left\|g_{\varepsilon}^{+}\right\|_{2} .
\end{aligned}
$$

From $|a|_{\infty}<\frac{4 \pi^{2}}{T^{2}}$ for $\varepsilon>0$ sufficiently small, there exists a positive constant $M_{1}^{\prime}$ such that

$$
\int_{0}^{T}\left|x^{\prime}(t)\right|^{2} d t \leq M_{1}^{\prime}
$$

From (2.6) and by applying Hölder's inequality, we have

$$
|x|_{\infty} \leq D_{2}+\int_{0}^{T}\left|x^{\prime}(s)\right| d s \leq D_{2}+\sqrt{T}\left(\int_{0}^{T}\left|x^{\prime}(s)\right|^{2} d s\right)^{\frac{1}{2}} \leq D_{2}+\sqrt{T} M_{1}^{\prime \frac{1}{2}}=M_{1} .
$$

On the other hand, from $x(0)=x(T)$, we know that there is a point $t_{1} \in[0, T]$ such that $x^{\prime}\left(t_{1}\right)=0$, and then $\left|x^{\prime}(t)\right|=\left|x^{\prime}\left(t_{1}\right)+\int_{t_{1}}^{t} x^{\prime \prime}(s) d s\right| \leq \int_{0}^{T}\left|x^{\prime \prime}(s)\right| d s$. From (2.3) and (2.8), we 
have

$$
\begin{aligned}
\left|x^{\prime}\right|_{\infty} & \leq \int_{0}^{T}\left|x^{\prime \prime}(t)\right| d t \\
& \leq \lambda \int_{0}^{T}|a(t)||x(t)| d t+\lambda \int_{0}^{T} \frac{1}{x(t)} d t \\
& \leq \lambda|a|_{\infty} M_{1} T+\lambda \int_{0}^{T}\left(\varepsilon x(t)+g_{\varepsilon}^{+}(t)\right) d t \\
& \leq \lambda|a|_{\infty} M_{1} T+\lambda \varepsilon M_{1} T+\lambda \sqrt{T}\left(\int_{0}^{T}\left|g_{\varepsilon}^{+}(t)\right|^{2} d t\right)^{\frac{1}{2}} \\
& \leq \lambda|a|_{\infty} M_{1} T+\lambda \varepsilon M_{1} T+\lambda \sqrt{T}\left\|g_{\varepsilon}^{+}\right\|_{2}:=\lambda M_{2},
\end{aligned}
$$

i.e.,

$$
\left|x^{\prime}\right|_{\infty} \leq \lambda M_{2}
$$

Multiplying (2.3) by $x^{\prime}(t)$, we get

$$
x^{\prime \prime}(t) x^{\prime}(t)+\lambda a(t) x(t) x^{\prime}(t)=\lambda \frac{x^{\prime}(t)}{x(t)} .
$$

Let $\tau \in[0, T]$ be as in (2.5). For any $\tau \leq t \leq T$, we integrate (2.12) on $[\tau, t]$ and get

$$
\begin{aligned}
\lambda \int_{x(\tau)}^{x(t)} \frac{1}{u} d u & =\lambda \int_{\tau}^{t} \frac{x^{\prime}(s)}{x(t)} d s \\
& =\frac{1}{2} x^{\prime}(t)^{2}-\frac{1}{2} x^{\prime}(\tau)^{2}+\lambda \int_{\tau}^{t} a(s) x(s) x^{\prime}(s) d s
\end{aligned}
$$

By (2.11) we have

$$
\begin{aligned}
& x^{\prime}(t)^{2} \leq \lambda^{2} M_{2}^{2}, \\
& \left|\int_{\tau}^{t} a(s) x(s) x^{\prime}(s) d s\right| \leq \lambda|a|_{\infty} M_{1} M_{2} T .
\end{aligned}
$$

With these inequalities we can derive from (2.13) that

$$
\left|\int_{x(\tau)}^{x(t)} \frac{1}{u} d u\right| \leq M_{2}^{2}+|a|_{\infty} M_{1} M_{2} T .
$$

So, we know that there exists $M_{3}>0$ such that

$$
x(t) \geq M_{3}, \quad \forall t \in[\tau, T]
$$

since $\lim _{x \rightarrow 0^{+}} \int_{1}^{x} \frac{1}{u} d u=+\infty$. The case $t \in[0, \tau]$ can be treated similarly.

Having in mind (2.5), (2.10), (2.11) and (2.15), we define

$$
\Omega=\left\{x \in C_{T}^{1}: E_{1}<x(t)<E_{2} \text { and }\left|x^{\prime}(t)\right|<E_{3} \forall t \in \mathbb{R}\right\},
$$


where $0<E_{1}<\min \left\{M_{3}, D_{1}\right\}, E_{2}>\max \left\{M_{1}, D_{2}\right\}$ and $E_{3}>M_{2}$. Then condition (i) of Lemma 2.1 is satisfied. For a constant $x \in \operatorname{ker} L, x>0$, we have

$$
\bar{g}(x):=\frac{1}{T} \int_{0}^{T}\left(a(t) x(t)-\frac{1}{x(t)}\right) d t .
$$

Obviously, $\bar{g}(x)<0$ for all $x \in\left(0, E_{1}\right), \bar{g}(x)>0$ for all $x>E_{2}$, so condition (ii) of Lemma 2.1 holds. Set

$$
H(x, \mu)=\mu x+(1-\mu) \frac{1}{T} \int_{0}^{T}\left(a(t) x(t)-\frac{1}{x(t)}\right) d t,
$$

we have $x H(x, \mu)>0$. Thus $H(x, \mu)$ is a homotopic transformation and

$$
\begin{aligned}
\operatorname{deg}\{F, \Omega \cap \mathbb{R}, 0\} & =\operatorname{deg}\left\{\frac{1}{T} \int_{0}^{T}\left(a(t) x(t)-\frac{1}{x(t)}\right) d t, \Omega \cap \mathbb{R}, 0\right\} \\
& =\operatorname{deg}\{x, \Omega \cap \mathbb{R}, 0\} \neq 0 .
\end{aligned}
$$

Thus assumption (iii) of Lemma 2.1 is also verified. Therefore (2.2) has at least one positive $T$-periodic solution.

Next, we apply Theorem 2.1 to Brillouin electron beam focusing system (1.1). Equation (1.1) is of the form (2.2) with $a(t)=a(1+\cos 2 t)$.

Theorem 2.2 If $a \in\left(0, \frac{1}{2}\right)$, then (1.1) has at least one positive $2 \pi$-periodic solution.

Proof If $a<\frac{1}{2}$, then

$$
|a|_{\infty}=2 a<1=\frac{4 \pi^{2}}{T^{2}}
$$

i.e., $|a|_{\infty}<\frac{4 \pi^{2}}{T^{2}}$ holds. Theorem 2.1 implies that (1.1) has at least one $2 \pi$-periodic positive solution.

Finally, we present an example to illustrate our result.

Example 2.1 Consider the second order differential equation with singularity:

$$
x^{\prime \prime}(t)+(1+\cos t)=\frac{1}{x} .
$$

It is clear that $T=\pi, a(t)=1+\cos t$. Obviously,

$$
|a|_{\infty}=\max _{t \in[0, T]}|1+\cos t|=2<4=\frac{4 \pi^{2}}{\pi^{2}} .
$$

Therefore, (2.17) has at least one $\pi$-periodic solution by application of Theorem 2.1. 
Authors' contributions

ZBC and SWY worked together in the derivation of the mathematical results. Both authors read and approved the final manuscript.

\section{Acknowledgements}

ZBC and SWY would like to thank the referee for invaluable comments and insightful suggestions. This work was supported by the National Natural Science Foundation of China (No. 11501170), China Postdoctoral Science Foundation funded project (No. 2016M590886), Fundamental Research Funds for the Universities of Henan Province (NSFRF140142), Henan Polytechnic University Outstanding Youth Fund (J2015-02) and Henan Polytechnic University Doctor Fund (B2013-055).

\section{Publisher's Note}

Springer Nature remains neutral with regard to jurisdictional claims in published maps and institutional affiliations.

Received: 13 February 2017 Accepted: 28 April 2017 Published online: 15 May 2017

\section{References}

1. Bevc, V, Palmer, J, Süsskind, C: On the design of the transition region of axisymmetric, magnetically focused beam valves. J. Br. Inst. Radio Eng. 18, 696-708 (1958)

2. Ding, T: Applications of Qualitative Methods of Ordinary Differential Equations. Higher Education Press, BeiJing (2004)

3. Ding, T: A boundary value problem for the periodic Brillouin focusing system. Acta Sci. Natur. Univ. Pekinensis 11, 31-38 (1965)

4. Ye, Y, Wang, X: Nonlinear differential equations in electron beam focusing theory. Acta Math. Appl. Sin. 1, 13-41 (1978)

5. Zhang, M: Periodic solutions of Liénard equations with singular forces of repulsive type. J. Math. Anal. Appl. 203, 254-269 (1996)

6. Torres, P: Existence and uniqueness of elliptic periodic solutions of the Brillouin electron beam focusing system. Math. Methods Appl. Sci. 23, 1139-1143 (2000)

7. Garrione, M, Zamora, M: Periodic solutions of the Brillouin electron beam focusing equation. Commun. Pure Appl. Anal. 13, 961-975 (2014)

8. Manásevich, R, Mawhin, J: Periodic solutions for nonlinear systems with p-Laplacian-like operator. J. Differ. Equ. 145, 367-393 (1998)

9. Xin, Y, Cheng, Z: Positive periodic solution of $p$-Laplacian Liénard type differential equation with singularity and deviating argument. Adv. Differ. Equ. 2016, 41 (2016)

10. Torres, $P, C$ Cheng, Z, Ren, J: Non-degeneracy and uniqueness of periodic solutions for $2 n$-order differential equations. Discrete Contin. Dyn. Syst., Ser. A 33, 2155-2168 (2013)

\section{Submit your manuscript to a SpringerOpen ${ }^{\circ}$ journal and benefit from:}

- Convenient online submission

Rigorous peer review

- Immediate publication on acceptance

- Open access: articles freely available online

- High visibility within the field

- Retaining the copyright to your article 